\title{
Characterization of the archaeal community fouling a membrane bioreactor
}

\author{
Jinxue Luo ${ }^{1,2,3}$, Jinsong Zhang ${ }^{4}$, Xiaohui Tan ${ }^{3}$, Diane McDougald ${ }^{2,5,6}$, Guoqiang Zhuang ${ }^{1}$, \\ Anthony G. Fane ${ }^{4}$, Staffan Kjelleberg ${ }^{2,5,6}$, Yehuda Cohen ${ }^{2,6}$, Scott A. Rice R,5,6,* $^{2}$
}

1. Key Laboratory of Environmental Biotechnology, Research Center for Eco-Environmental Sciences, Chinese Academy of Sciences, Beijing 100085, China. E-mail: luojinxue@163.com

2. School of Biological Sciences, Nanyang Technological University, Singapore 639798, Singapore

3. Advanced Environmental Biotechnology Centre, Nanyang Environment and Water Research Institute, Nanyang Technological University, 639798, Singapore

4. Singapore Membrane Technology Centre, Nanyang Environment and Water Research Institute, Nanyang Technological University, 639798, Singapore

5. Centre for Marine Bio-Innovation, School of Biotechnology and Biomolecular Sciences, The University of New South Wales, Sydney, NSW 2052, Australia

6. Singapore Centre on Environmental Life Sciences Engineering, Nanyang Technological University, 639798, Singapore

\section{A R T I C L E I N F O}

\section{Article history:}

Received 4 April 2014

Revised 14 July 2014

Accepted 19 July 2014

Available online 5 January 2015

\section{Keywords:}

Biofouling

Biofilm formation

Biofilm microbial community

Archaeal community

Membrane bioreactor

\begin{abstract}
A B S T R A C T
Biofilm formation, one of the primary causes of biofouling, results in reduced membrane flux or increased transmembrane pressure and thus represents a major impediment to the wider implementation of membrane bioreactor (MBR) technologies for water purification. Most studies have focused on the role of bacteria in membrane fouling as they are the most dominant and best studied organisms present in the MBR. In contrast, there is limited information on the role of the archaeal community in biofilm formation in MBRs. This study investigated the composition of the archaeal community during the process of biofouling in an MBR. The archaeal community was observed to have lower richness and diversity in the biofilm than the sludge during the establishment of biofilms at low transmembrane pressure (TMP). Clustering of the communities based on the Bray-Curtis similarity matrix indicated that a subset of the sludge archaeal community formed the initial biofilms. The archaeal community in the biofilm was mainly composed of Thermoprotei, Thermoplasmata, Thermococci, Methanopyri, Methanomicrobia and Halobacteria. Among them, the Thermoprotei and Thermoplasmata were present at higher relative proportions in the biofilms than they were in the sludge. Additionally, the Thermoprotei, Thermoplasmata and Thermococci were the dominant organisms detected in the initial biofilms at low TMP, while as the TMP increased, the Methanopyri, Methanomicrobia, Aciduliprofundum and Halobacteria were present at higher abundances in the biofilms at high TMP.

(c) 2014 The Research Center for Eco-Environmental Sciences, Chinese Academy of Sciences.
\end{abstract} Published by Elsevier B.V.

\footnotetext{
* Corresponding author. E-mail: RSCOTT@ntu.edu.sg (Scott A. Rice).
} 


\section{Introduction}

Membrane bioreactors (MBRs) represent a class of technology integrating the activated sludge mediated nutrient removal and membrane mediated sludge-water separation (Kraume and Drews, 2010), and have many advantages in the wastewater treatment process (WWTP) in comparison with the conventional gravity dependent WWTP. These advantages include a smaller treatment space required, less hydraulic retention time, less activated sludge biomass production and a higher quality of effluent (Melin et al., 2006; Li et al., 2013). However, all MBR systems suffer from biofouling which negatively impacts on MBR performance, resulting in higher operating costs (Le-Clech et al., 2006). Biofouling is the result of deposition of the microorganisms and microbial extracellular polymeric substances (EPS), e.g., polysaccharides and proteins, on the membrane surface. The microbes and their secreted EPS attach onto the membrane and proliferate to form a cake-layer on the membrane, leading to clogging of the membrane (Nguyen et al., 2012). Membrane clogging by the biofilm reduces the water permeability through the membrane, resulting in a lower permeate production (flux) or a higher transmembrane pressure (TMP) to maintain the constant flux.

Biofilm formation is a ubiquitous phenomenon for microorganisms in nature (Simões et al., 2010). The microbial community involved in the biofilm formation in MBR has been studied intensively. For example, bacterial communities including the Proteobacteria, Bacteroidetes and Actinobacteria, had been reported to compose the majorities of biofouling community in MBR (Miura et al., 2007; Ivnitsky et al., 2007). The Bacteroidetes and Firmicutes displayed a higher competence to live in the biofilm layers relative to the activated sludge (Lim et al., 2012). Additionally, fungal organisms, such as Saccharomyces sp. and Cryptococcus sp., are also present in both the anoxic and aerobic sludge and form biofilms on the MBR membranes (Ravi et al., 2009; Bojsen et al., 2012).

The archaea represent the third domain of life and exhibit considerable diversity. Indeed, this group of microorganisms is best known for their ability to adapt to extreme environments, such as the hot springs (Song et al., 2013), acidic environment (Edwards et al., 2000) and hypersaline lakes (Oren, 2001). They have even been identified in activated sludge from wastewater treatment plants (Gómez-Silván et al., 2010; Zhang et al., 2011). Some archaeal groups, e.g., Haloarchaea and Crenarchaea, were demonstrated to be capable of adhering to surfaces to form biofilms (Fröls et al., 2012; Koerdt et al., 2010). The quorum sensing (QS) signals, which regulate the biofilm formation processes of bacterial community, were also reported to coordinate the development of archaeal biofilm (Orell et al., 2013). The carboxylated acyl homoserine lactones (C-AHLs) were used in the QS system of the methanogenic euryarchaea (Zhang et al., 2012). This was different from the bacterial QS system, where the N-acyl-homoserine lactones were used as the signals in QS phenomenon (Krysciak et al., 2011). The biofilm archaeal community was diverged in different wastewater treatment processes. In an anoxic/aerobic submerged biofilter system, the archaea Methanobacteria and Methanomicrobia were observed to compose the majority of the biofilm community (Gómez-Silván et al., 2010). In a reverse osmosis membrane system, the Methanomicrobia were also the dominant archaea of the biofilms on membrane surface (Al Ashhab et al., 2014). Besides, the Thermoprotei, Methanopyri, and Thermoplasmata were prevailing in the membrane biofilms in the reverse osmosis membrane system (Al Ashhab et al., 2014). In a pilot-scale membrane-coupled upflow anaerobic sludge blanket bioreactor, the methanogenic archaea, e.g., Methanosarcinales and Methanospirillaceae, persistently composed of the biofouling microbial community, even the membranes were cleaned by the chemical reagents (Calderón et al., 2011). Despite these reports, there is still very little data on the association of archaea with biofilm formation on MBR membranes and their impact on MBR performance (Calderón et al., 2013). Since some archaeal groups, such as methanogenic archaea and ammonia oxidizing archaea, are very important in nitrogen and carbon removal processes and prevailing in wastewater treatment activated sludge (Fredriksson et al., 2012; Tabatabaei et al., 2010), it is necessary to study the community diversity of archaeal biofilm on membranes in order to control the biofouling phenomenon in MBR systems.

In this study, the archaeal communities on hollow fiber membranes and in activated sludge were characterized in a MBR through high-throughput sequencing of the 16S rRNA gene. The phylotypes of archaea were determined correlating with the increase in TMP.

\section{Materials and methods}

\subsection{MBR set-up and operation}

A laboratory scale anoxic/oxic membrane bioreactor (A/O MBR) was conducted to treat the synthetic wastewater, which was composed of glucose $(320 \mathrm{mg} / \mathrm{L})$, beef extract $(60 \mathrm{mg} / \mathrm{L})$, peptone $(80 \mathrm{mg} / \mathrm{L}), \quad \mathrm{KH}_{2} \mathrm{PO}_{4}(7 \mathrm{mg} / \mathrm{L}), \quad \mathrm{MgSO}_{4} \cdot 7 \mathrm{H}_{2} \mathrm{O} \quad(14 \mathrm{mg} / \mathrm{L})$, $\mathrm{FeSO}_{4} \cdot 7 \mathrm{H}_{2} \mathrm{O}(7.3 \mathrm{mg} / \mathrm{L})$ and sodium acetate $(90 \mathrm{mg} / \mathrm{L})$. The total organic carbon (TOC) of synthetic wastewater was $200 \mathrm{mg} / \mathrm{L}$. The A/O MBR system was composed of an anoxic sludge tank, an aerobic sludge tank and a filtration tank in which a membrane module was installed (Fig. 1a). The membrane module was made with the hollow fiber PVDF membranes (ZeeWeed, GE, Singapore) and designed to be a "curtain" mode, where one end of the membrane piece was sealed and hung down into the sludge tank (free end), while the other end of the membrane was open and sealed into a collection chamber that was linked to the suction pump (Fig. 1a). The activated sludge was collected from the Ulu Pandan wastewater treatment plant in Singapore and acclimated in synthetic wastewater for 60 days before the start of the experiment. The concentrations of dissolved oxygen (DO) in the anoxic tank and aerobic tank were 0.1-0.2 mg/L and 5-6 mg/L, respectively. A peristaltic pump was used to re-circulate the liquor sludge from the filtration tank to anoxic tank. The mixed liquor suspended sludge (MLSS) was maintained at 6-8 g/L and 3-5 g/L in the anoxic tank and aerobic tank respectively. The MBR was operated with the constant flux, $15 \pm 1 \mathrm{~L} /\left(\mathrm{m}^{2} \cdot \mathrm{hr}\right)(\mathrm{LMH})$, at the room temperature of $25-26^{\circ} \mathrm{C}$. The hydraulic retention time (HRT) and sludge retention time (SRT) for the MBR were approximately $10 \mathrm{hr}$ and 25 days respectively. The parameters, such as membrane flux, TMP, pH, DO and temperature, were monitored and automatically recorded using a data logger and a computer. The TOC of the influent and permeate was measured using a multi N/C® 2100s (Analytik Jena AG, Thuringia, Germany).

\subsection{DNA extraction}

DNA of the biofilms and activated sludge was separately extracted by a modified CTAB-PEG protocol (Paithankar and Prasad, 1991; Griffiths et al., 2000). The biofilm and sludge samples were collected at two time points of low TMP stage (7 and $10 \mathrm{kPa}$ ) and two time points of high TMP stage (30 and $60 \mathrm{kPa}$ ). The mixed liquor suspended sludge was collected in microfuge tubes containing lysing matrix (FastPrep Kit, MP 

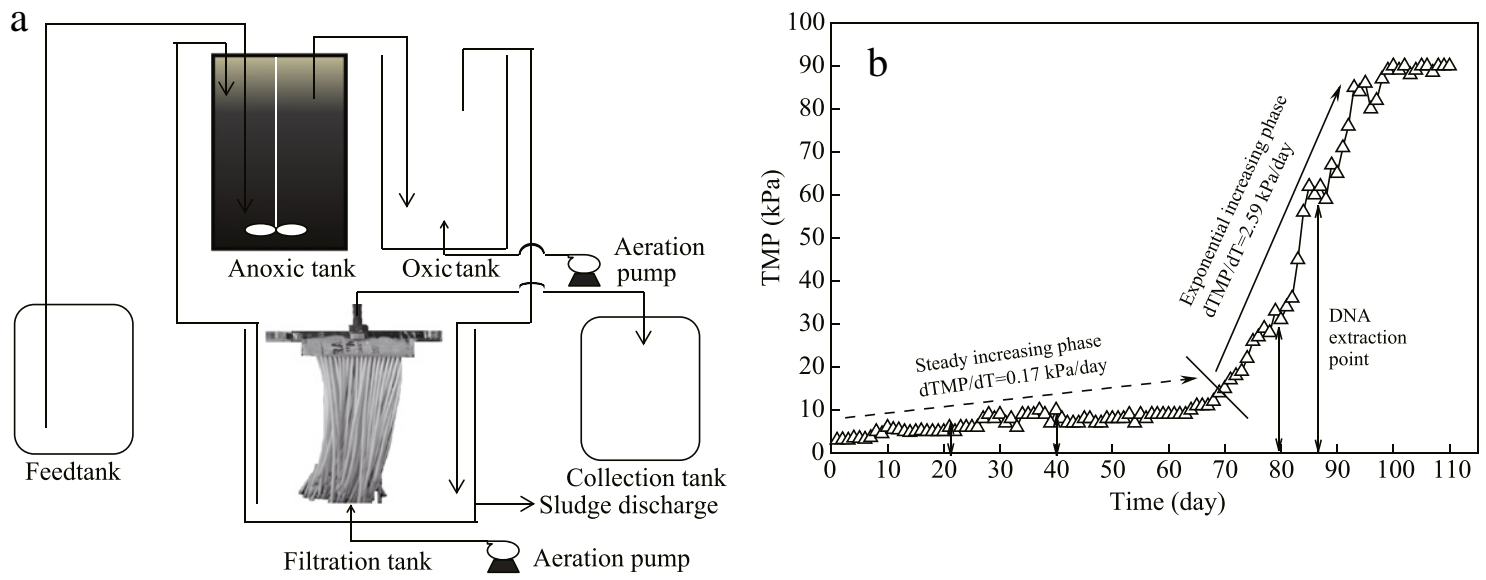

Fig. 1 - Schematic diagram and transmembrane pressure (TMP) increasing profile for the membrane bioreactor (MBR) system in this study. (a) The configuration of the MBR, where a "curtain" mode membrane module is installed in the filtration tank. (b) The TMP increasing profile. The triangles show the average TMP values in each day. The arrow with dashed line shows the period of TMP steady increasing phase and the arrow with solid line shows the period of TMP exponentially increasing phase. The lines with double arrows point to the time and TMP when the DNA was extracted.

Biomedicals, Singapore) by centrifugation at $17,000 \times g$ for $5 \mathrm{~min}$. To collect the biofilms, the fouled membrane was cut into small pieces and put into the MP lysing tubes (FastPrep Kit, MP Biomedicals, Singapore). Subsequently, the sample tubes were filled with $0.5 \mathrm{~mL}$ of $5 \%$ CTAB lysis solution and $0.5 \mathrm{~mL}$ phenol/chloroform/isoamyl alcohol (25:24:1 in volume ratio) and shaken in a Fast-Prep bead beater (FastPrep-24, MP Biomedicals, Singapore) at a speed of $5.5 \mathrm{~m} / \mathrm{sec}$ for $30 \mathrm{sec}$. After centrifugation at 17,000 $\times$ g for $5 \mathrm{~min}$, the top DNA solution was treated with RNase (final concentration: $10 \mu \mathrm{g} / \mathrm{mL}$ ) at $37^{\circ} \mathrm{C}$ for $30 \mathrm{~min}$. The DNA solution was then mixed with $0.5 \mathrm{~mL}$ chloroform/isoamyl alcohol (24:1 in volume ratio) and centrifuged at $17,000 \times g$ for $5 \mathrm{~min}$ to remove the protein contaminants. The clean DNA solution was mixed with 2 volumes of a $30 \%$ PEG solution and incubated at $4^{\circ} \mathrm{C}$ overnight to precipitate the DNA. After being centrifuged at $17,000 \times g$ for $15 \mathrm{~min}$, the DNA pellets were washed with $70 \%$ ice cold ethanol 3 times. The DNA pellets were air-dried and dissolved in pure water. The DNA solution was quantified using a NanoDrop spectrophotometer (Thermo Scientific, Singapore) and stored at $-80^{\circ} \mathrm{C}$.

\subsection{Sequencing of DNA and sequence process}

The DNA samples were sequenced using the " 454 " pyrosequencing platform (Research and Testing Laboratory, Texas, US) (Handl et al., 2011). The primers selected for PCR of the archaeal community were Arch349F (5'-GYGCASCAGKCGMGAAW-3') and Arch806R (5'-GGACTACVSGGGTATCTAAT-3') (Takai and Horikoshi, 2000). The average number of sequence reads for every sample was approximately $5800 \pm 3500$. All DNA sequences had been deposited to the Sequence Read Archive of NCBI. The accession No. of sequence data packets are SRR1201383, SRR1201385-SRR1201387, SRR1201389-SRR1201400.

The sequence data was processed using the MOTHUR software based on the Costello analysis pipeline (Costello et al., 2009; Schloss et al., 2009). The barcodes, primers, and low quality sequences (below 25) were removed by "Trim.seqs". The size of sequence package was reduced through the "unique.seqscluster.fragments". The unique sequences were aligned against the Silva archaeal reference using the "align.seqs" and further removed the error bases and nonsense columns through the process of "screen.seqs-filter.seqs". The sequence package was denoised using "pre.cluster" to merge the rare sequences differing by less than 2 bases to more abundant sequences. The chimeric sequences were checked and removed from the datasets using the "chimera.slayer-remove.seqs". The final sequences were assigned to taxonomic groups based on the SILVA archaeal reference using the "classify.seqs" (Pruesse et al., 2007). The criteria for the sequence classification (similarity to the reference sequence) were Species (>97\%), Genus (94\%-97\%), Family (90\%-94\%), Order (85\%-90\%), Class (80\%-85\%), and Phylum (75\%-80\%) (Lim et al., 2012). Sequences below the similarity criteria were classified into unidentified groups at each taxonomic rank.

\subsection{Phylotype-based community analysis}

A shared phylotype file was generated through the command "make.shared" for all the samples. The relationship between the archaeal phylotypes and sequences was calculated by MOTHUR using the command of "summary.single". Clustering trees and Nonmetric Multidimensional Scaling (NMDS) plot were created between the samples based on the group average Bray-Curtis similarity of phylotype compositions in the different groups (Clarke, 1993). The similarity or dissimilarity between the samples was calculated by the operation "SIMPER" in Primer 6 (PRIMER-E Ltd, Lutton, UK) (Clarke, 1993). The contributions of the phylotypes to the similarity or dissimilarity were calculated based on the relative abundances of phylotypes between the samples.

\subsection{Statistical analysis}

The significance of community difference was examined by the Student's T-test in SPSS (version 16.0, IBM, Singapore). 
The differences of archaeal community between the activated sludge and biofilms were significant at $p<0.05$ (Willett et al., 2004). The variations of archaeal community in biofilms from low TMP to high TMP were significant at $p<0.05$ (Willett et al., 2004).

\section{Results}

\subsection{Biofouling phenomenon and TMP increase in A/O MBR}

During the MBR operation, the TOC removal efficiency was in the range of $95 \%-98 \%$. The biofouling behavior, as measured by changes in TMP, was monitored over a 110 day period. The increase in TMP is generally associated with increased biomass of biofilm on membrane surface. The TMP profile was consisted of two phases, the steady increasing phase and the exponentially increasing phase (Fig. 1b). The TMP steady increasing phase was represented by the first 70 days of operation where the TMP increased from 3 to $15 \mathrm{kPa}$. During exponentially increasing phase, the TMP increased dramatically over the subsequent 29 days from 15 to $90 \mathrm{kPa}$. The rate of TMP increase was $0.17 \mathrm{kPa} /$ day and $2.59 \mathrm{kPa} /$ day in the two phases, respectively. To determine if archaea were associated with membrane fouling and if there were community shifts that correlated with the two phases of operation, DNA was extracted from specific time points $(7,10,30$ and $60 \mathrm{kPa})$ and sequenced using archaeal specific 16 s rRNA gene primers.

\subsection{Diversity of archaeal community in biofilm and sludge}

After the sequence processing and assignment, a total of 24 Classes of archaea were classified based on the 27,300 archaeal sequences in the biofilm and sludge samples. The coverage of archaeal community at Class level was $98 \%-99 \%$ in the biofilm and sludge. The diversity indices were investigated for the archaeal community in biofilm and sludge, including Margalef's richness index and Shannon diversity index that combined the richness and evenness together.

At low TMP (7 kPa), the biofilms were found to have both lower Margalef's index (1.85) and Shannon index (0.98) in comparison with the sludge (2.11 in Margalef's index and 1.72 in Shannon index) (Fig. 2), indicating that only a subset of the sludge archaeal community was found in the low pressure biofilms. When the TMP increased from 10 to $60 \mathrm{kPa}$, the diversity and richness of the archaea in the biofilm increased. At $60 \mathrm{kPa}$, the richness of archaeal community became almost the same in the high pressure biofilm (2.509) and sludge (2.512) while the Shannon diversity index was lower in the biofilm (1.58) than in sludge (1.72).

\subsection{Relationship of archaeal communities in biofilms and activated sludge}

The Bray-Curtis similarity was calculated to study the relationship of archaeal communities in the sludge and biofilms. To facilitate the analysis, the similarity tree and NMDS plot were constructed.
The archaeal communities in initial biofilms were observed to diverge from the sludge communities at $7 \mathrm{kPa}$, showing $41.7 \%$ of dissimilarity between the two types of archaeal communities (Fig. 3a). However, when the TMP increased to $30-60 \mathrm{kPa}$, the archaeal community showed a closer relationship between the biofilm and sludge, which had a lower dissimilarity at $30 \mathrm{kPa}$ (17.6\% dissimilarity) and $60 \mathrm{kPa}$ (13.1\% dissimilarity). This was apparent based on the NMDS plot, where the archaeal communities in the early biofilms and sludge samples at $7 \mathrm{kPa}$ were divided into two different clusters while the archaeal communities at 30-60 kPa were clustered into one group for the late biofilms and sludge (Fig. 3b). This indicated that the early biofilms were established by the archaeal community which had different compositions from the sludge community while the archaeal communities in the late biofilms and sludge at high TMP became more similar again.

It was also noted that the archaeal community changed in biofilms as the TMP increased. In comparison with the biofilm community at $7 \mathrm{kPa}$, the archaeal communities displayed dissimilarities of $49.5 \%, 46.2 \%$ and $48.5 \%$ in biofilms at 10,30 and $60 \mathrm{kPa}$ respectively (Fig. 3a). The archaeal biofilm communities at high TMP (30-60 kPa) were more similar with each other relative to the archaeal communities in biofilms at low TMP (7-10 kPa) (Fig. 3b). This was an indication that the archaeal communities had distinct compositions in biofilms in low and high TMP phases.

\subsection{Composition of the archaeal communities at low TMP}

\subsubsection{Archaea in seed sludge}

When the seed sludge, which was used to inoculate the reactors, was examined, a total of 19 Classes of archaea were identified at $7 \mathrm{kPa}$. Exploration of the community composition structure in detail revealed that the archaeal community was dominated by Thermoprotei (45.2\% in abundance), Thermococci $(21.58 \%$ in abundance), Nanoarchaeum (9.9\% in abundance), Candidatus Korarchaeum (6.47\% in abundance) and Methanococci (6.36\% in abundance), which represented $89.5 \%$ of the archaeal community in the seed sludge (Fig. 4).

\subsubsection{Archaea in early biofilm}

In the biofilms formed at $7 \mathrm{kPa}$, a total of 19 Classes of archaea were classified. Among them, 5 Classes of archaea were observed to predominate in the whole community, including Thermoprotei (67.31\% in abundance), Thermoplasmata (21.49\% in abundance), Thermococci (2.58\% in abundance), Methanococci $(2.2 \%$ in abundance) and Methanopyri (1.09\% in abundance). These organisms comprised $94.7 \%$ of the archaeal community in the low pressure biofilms (Fig. 4).

2.4.3. Comparison of the archaeal communities in sludge and biofilm at low TMP

The NMDS plot in Fig. 3b showed that the archaeal community of the biofilm differed from the sludge at low TMP. The major differences were attributed to two groups of archaea. The first group included the Thermoprotei and Thermoplasmata, which were enriched in the early biofilms rather than the sludge (Fig. 5). The Thermoprotei were the most abundant archaea in 

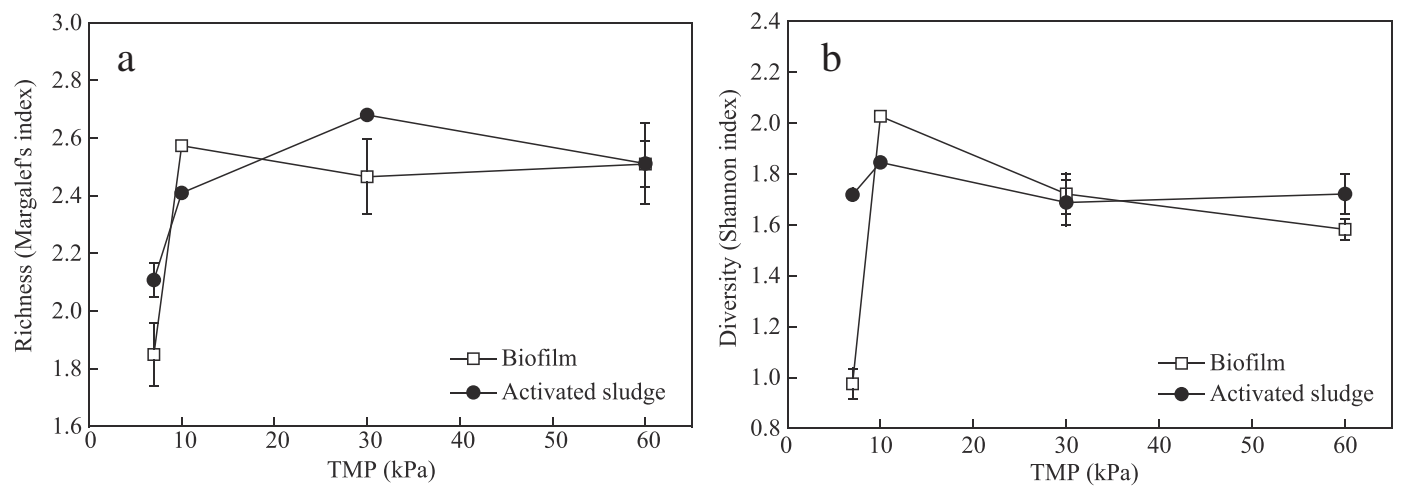

Fig. 2 - Diversity of archaeal community in biofilm and sludge in the TMP increasing process. (a) Richness of community, indicated by the Margalef's index; (b) diversity of archaeal community, indicated by the Shannon index.

both the sludge and biofilms at $7 \mathrm{kPa}$. But the abundance of Thermoprotei was higher in biofilm (67.3\%) relative to the sludge $(45.2 \%)$ ( $p=0.026$ by $\mathrm{T}$ test). A similar phenomenon was also observed for the Thermoplasmata, which only accounted for $2.37 \%$ of the sludge archaeal community but were highly enriched in the low pressure biofilms (21.5\% in abundance) ( $p=0.021$ by $\mathrm{T}$ test).

Another interesting group included 7 Classes of archaea (Fig. 5), which had higher abundances in the sludge relative to the early biofilms. This was apparent for the Thermococci and Nanoarchaeum, which accounted for $21.6 \%$ and $9.9 \%$ in the sludge but only had abundances of $2.6 \%$ and $1 \%$ in the biofilms ( $p=0.0001$ and 0.002 respectively by $\mathrm{T}$ test). Similar variation was observed for Candidatus Korarchaeum (6.47\% in sludge us. $1.2 \%$ in biofilm, $p=0.001$ by $T$ test) and Methanococci (6.36\% in sludge vs. $2.2 \%$ in biofilm, $p=0.011$ by $T$ test). It was noted that these 4 Classes of archaea were still the dominant archaeal groups in the biofilms ( $>1 \%$ ), even though they did not have as high abundance as in sludge.

\subsection{Composition of archaeal communities at high TMP}

\subsubsection{Archaea in sludge at high TMP}

When the TMP jumped from low $(7 \mathrm{kPa})$ to high pressure (30-60 kPa), the sludge archaeal community was dominated by the Thermoprotei (34.1\%), Methanopyri (33.7\%), Methanomicrobia (13\%), Candidatus Korarchaeum (7.13\%) and Methanococci (3.18\%), which composed $91 \%$ of archaeal community (Fig. 4). Compared to the low TMP sludge community, Thermoprotei were still the dominant group in the sludge while the Methanopyri and Methanomicrobia increased dramatically from $1.4 \%$ to $33.7 \%$ and $2.6 \%$ to $13 \%$ respectively. Meanwhile, the Thermococci and Nanoarchaeum decreased the abundance in sludge, $21.6 \%$ to $0.9 \%$ and $9.9 \%$ to $1.8 \%$, respectively.

\subsubsection{Archaea in late biofilms at high TMP}

As indicated by the NMDS plot (Fig. 3b), the late biofilms had a similar community composition with the sludge at high TMP. Specifically, the major archaea in the high pressure biofilms

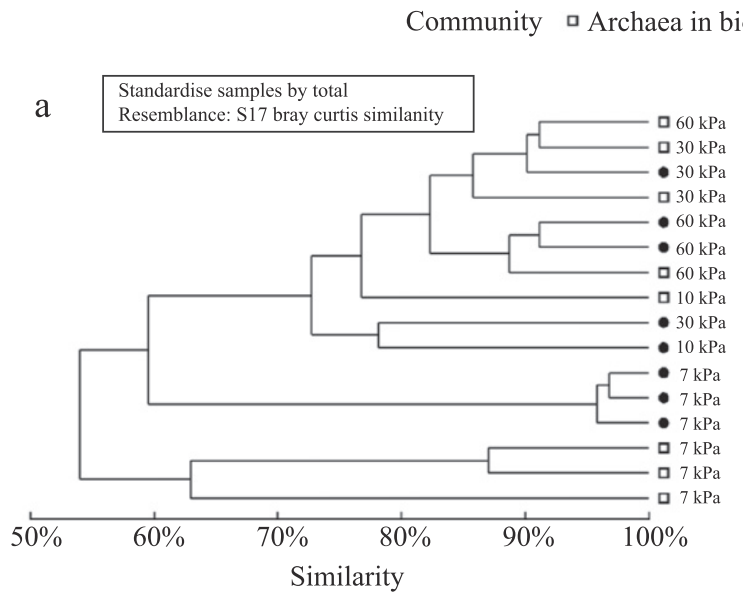

$b$

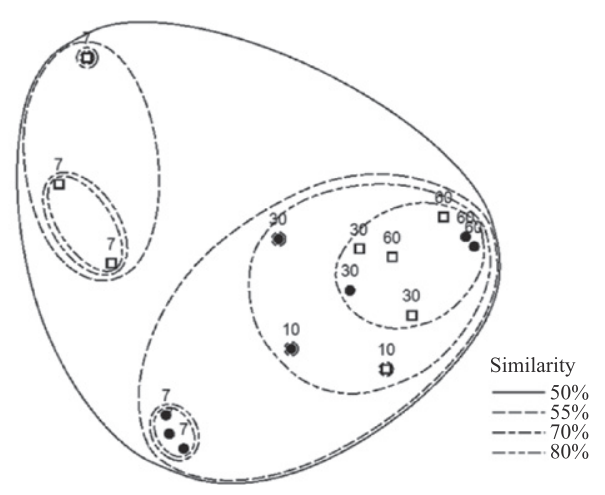

Fig. 3 - Relationship of archaeal communities in biofilms and sludge during the change in TMP during operation of a laboratory scale MBR. (a) Clustering tree of the archaeal communities was based on the Bray-Curtis similarity matrix; (b) NMDS plot for the archaeal communities was based on the Bray-Curtis similarity index. The numbers in the NMDS plot represent the TMP values $(\mathrm{kPa})$ when the biofilm and sludge samples were collected. 


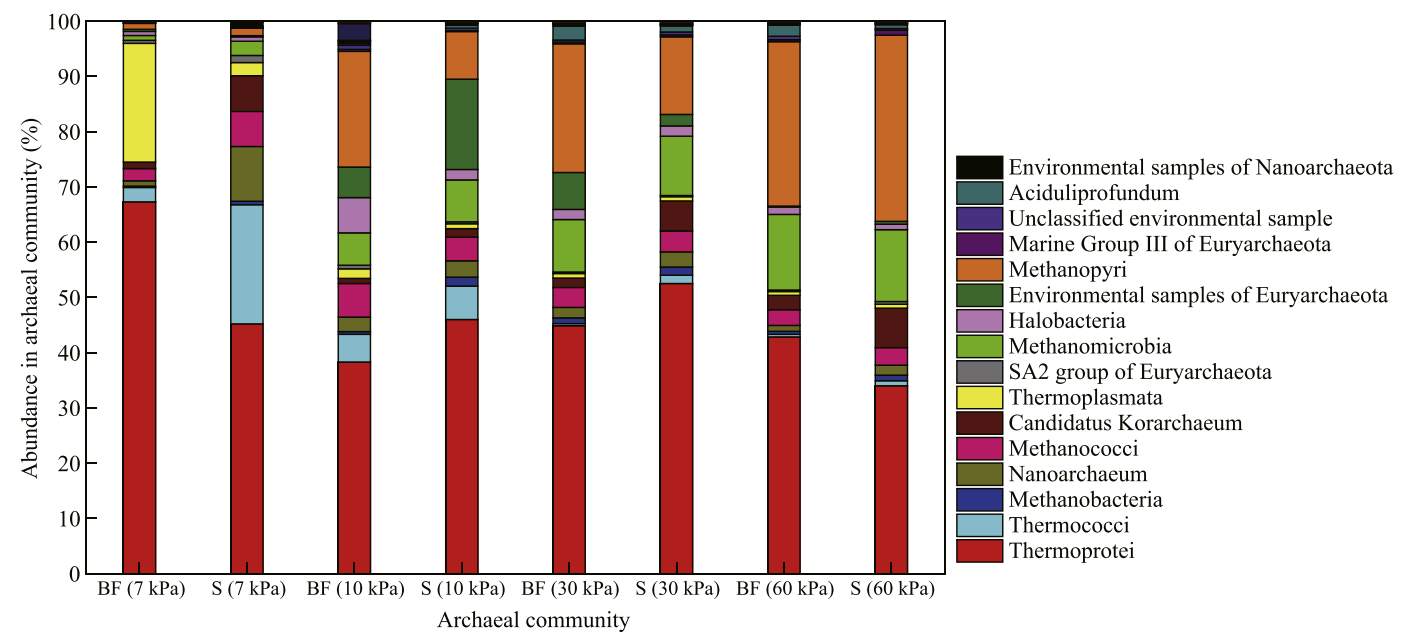

Fig. 4 - Archaeal community in the sludge and biofilm at different TMP values during operation of the MBR. The abundances were the average percentage of each archaeal group in the replicated samples. The labels "BF" and " $\mathrm{S}$ " represent the biofilm and sludge respectively. The pressures in the parentheses indicate the TMP values when the biofilm and sludge samples were collected.

included the Thermoprotei (42.9\%), Methanopyri (29.8\%), Methanomicrobia (13.69\%), Methanococci (2.87\%) and Candidatus Korarchaeum (2.61\%), which comprised $91.8 \%$ of the whole archaeal community. These dominant archaeal groups were quite the same with the major groups of sludge archaeal community at high TMP (Fig. 4).

\subsection{Variation of archaeal community in biofilms as the TMP} increased

Based on the NMDS plot (Fig. 3), it was shown that the structure of archaeal biofilm community changed as the TMP increased. In comparison with the archaeal biofilm community at low

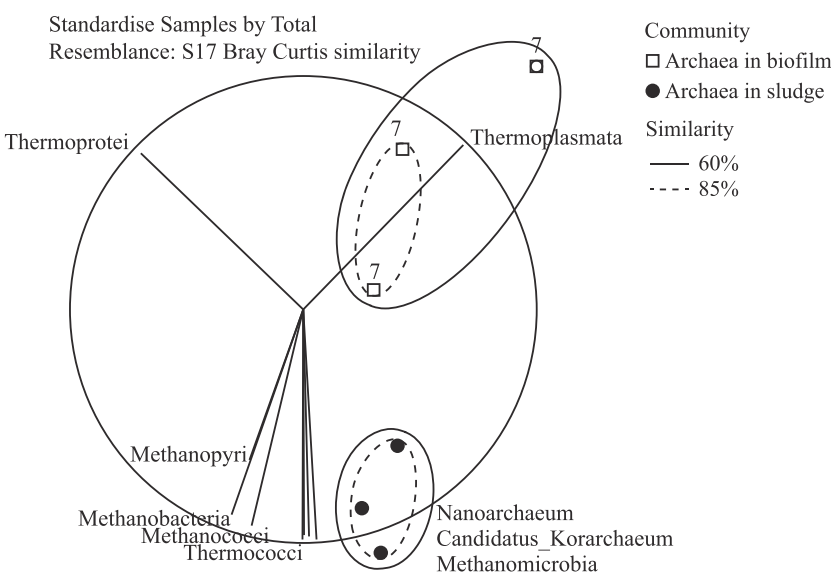

Fig. 5 - Major differences of archaeal communities between biofilms and sludge at low TMP. The NMDS plot is based on the Bray-Curtis similarity of the archaeal communities. The numbers in the NMDS plot represent the TMP values (kPa) when the biofilm and sludge samples were collected. The line branches point to the key organisms that had higher abundance in biofilms or sludge.
TMP $(7 \mathrm{kPa})$, some archaea were observed to increase in abundance in the biofilms when the TMP jumped to a high level (30-60 kPa), such as Methanopyri, Methanomicrobia, Aciduliprofundum, Halobacteria and Candidatus Korarchaeum (Fig. 6). The Methanopyri and Methanomicrobia were both the rare archaea in early biofilms, $1.1 \%$ and $0.9 \%$ in abundance respectively. However, the abundance increased much higher for the Methanopyri (29.8\%, $p=0.0001$ by $\mathrm{T}$ test) and Methanomicrobia (13.7\%, $p=0.001$ by $\mathrm{T}$ test) at $30-60 \mathrm{kPa}$, making them the majority of archaeas in the high pressure biofilms (Fig. 4). This phenomenon was also observed for the Aciduliprofundum $(0.05 \%$ at $7 \mathrm{kPa}$ us. $2.1 \%$ at $30-60 \mathrm{kPa}, p=$ 0.029 by $\mathrm{T}$ test) and Halobacteria (0.74\% at $7 \mathrm{kPa}$ us. $1.34 \%$ at 30 $60 \mathrm{kPa}, p=0.039$ by $\mathrm{T}$ test).

Meanwhile, 3 Classes of archaea decreased in biofilm abundance as the TMP increased, which were the Thermoprotei, Thermoplasmata and Thermococci (Fig. 6). The Thermoprotei accounted for $67.3 \%$ of the biofilm archaeal community at the low pressure but had a lower abundance $(42.9 \%, p=0.002$ by $\mathrm{T}$ test) in biofilms at high pressure. The other 2 Classes of archaea were present at much lower abundances in the high pressure biofilms. For example, the Thermoplasmata decreased from $21.5 \%$ in low pressure biofilms to $0.7 \%$ in the high pressure biofilms ( $p=0.019$ by $\mathrm{T}$ test). A similar trend was observed for Thermococci $(2.58 \%$ at $7 \mathrm{kPa}$ us. $0.5 \%$ at $30-60 \mathrm{kPa}, p=0.01$ by $\mathrm{T}$ test).

\section{Discussion}

Biofilm formation on membranes is fundamental to the process of membrane fouling in MBRs (Miura et al., 2007). The archaeal community composition was determined for the sludge and membrane associated biofilms in this study. The most abundant biofilm associated archaea included the Methanomicrobia, Methanopyri, Thermoplasmata and Thermococci and Thermoprotei. This was consistent with 
Standardise samples by total resemblance: S17 Bray Curtis similarity
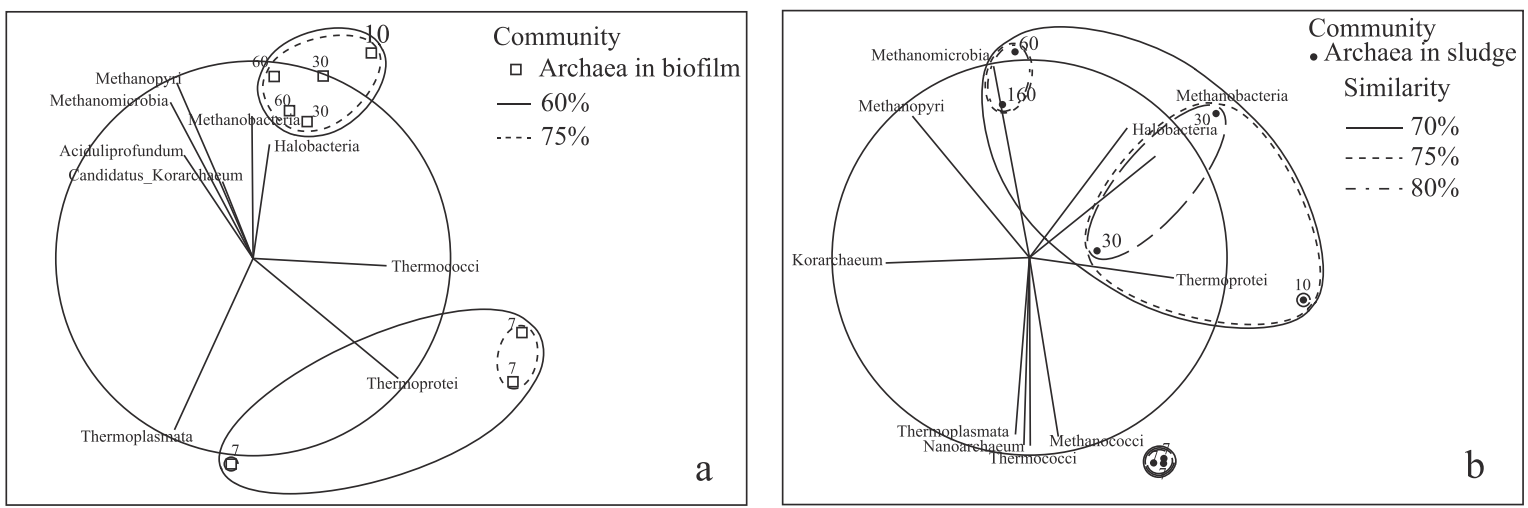

Fig. 6 - Major variations of archaeal community in the biofilm and sludge in the TMP increasing process. (a) Archaeal community in biofilm; (b) archaeal community in sludge. The NMDS plot is based on the Bray-Curtis similarity of the archaeal communities at different TMP. The numbers in the NMDS plot represent the TMP values (kPa) when the samples were collected. The line branches point to the key organisms that had higher contributions to biofilms or sludge at different TMP phases.

the previous studies (Calderón et al., 2011; Al Ashhab et al., 2014), indicating that these organisms may be the universal biofilm associated archaea in the membrane-based water purification processes. In comparison with the sludge community, the archaeal communities in biofilms formed a distinct community cluster at low TMP (Fig. 3). This indicated that a subset of sludge archaeal community may be selected during the early stage of biofilm formation on membrane. This was consistent with the bacterial community in biofilm formation where a subset of bacteria was selected from the sludge to form the initial biofilm in MBR (Piasecka et al., 2012). This finding was also supported by the different richness and Shannon diversity of the archaeal communities between the biofilms and sludge at $7 \mathrm{kPa}$ in this study. Thus, some archaeal groups in sludge, such as Thermoprotei and Thermoplasmata, may be better able to colonize onto the membrane during the initial stage of biofilm development. In previous studies, the Thermoprotei and Thermoplasmata have also been detected to form the biofilms in many different environmental sites, such as sulfide-rich cave wall (Macalady et al., 2007), acid mine drainage system (Justice et al., 2012) and wastewater pipe system (Gomez-Alvarez et al., 2012).

The structure of the archaeal biofilm community was found to change as the TMP increased during the operation of MBR. The Methanopyri, Methanomicrobia, Aciduliprofundum and Halobacteria increased their relative abundances in biofilm while the Thermoprotei, Thermoplasmata and Thermococci decreased the percentage as the TMP increased from 7 to $30-60 \mathrm{kPa}$, indicating that the former 3 archaeal groups may contribute more to the establishment of the initial biofilm while the latter 4 groups contribute more to the formation of late biofilm. This community temporal change was also reported in the previous study of the oral biofilms, dental plaque (Kolenbrander, 2000), indicating that it may be a common phenomenon that the biofilm formation by multiple species follows a particular pattern or successional process. While it is not possible to separate if the change in the archaeal community causes the increase in TMP or if it is an effect of the TMP rise, it does suggest that the archaeal community could be an important factor in the biofouling process of MBR.

It was also noted that the archaeal community of sludge and biofilms displayed similar community changes as the TMP increased. The richness and diversity of biofilm archaeal community increased as the TMP increased, resulting in similar richness and diversity at $30-60 \mathrm{kPa}$ for the biofilm and sludge communities. At 30-60 kPa, the biofilm archaeal community showed high similarity to the sludge community (80\%). This may indicate that the formation of biofilms by the initial archaeal residents facilitated the colonization of other sludge archaeal groups onto membranes. This phenomenon was also reported in one previous study, where the biofilms increased the adhesion of invertebrates to the biofouling layers (Zardus et al., 2008).

\section{Conclusions}

The archaeal community composition was investigated in activated sludge and membrane biofilms during the TMP increasing process of the MBR. The major biofilm associated archaea were the Methanomicrobia, Methanopyri, Methanococci, Thermoplasmata, Thermoprotei, and Thermococci. The Thermoprotei, Thermoplasmata and Thermococci may mainly contribute to the initial establishment of the biofilm, while the Methanopyri, Methanomicrobia, Aciduliprofundum and Halobacteria were the dominant archaea in the biofilms at high TMP. This succession of the archaeal community may be one of the reasons for the TMP exponential increase in MBR. Therefore, these results suggested that it would be valuable to better understand how these archaeal groups contribute to biofouling, in order to rationally develop approaches that can be used to alleviate the biofouling behavior through preventing the formation of the archaeal biofilms or dispersing the established archaeal biofilms. 


\section{Acknowledgments}

This research was supported by a research grant (MEWRC651/ 06/177) from the Environment and Water Industry Programme Office of Singapore. The authors also thank A/Prof Torsten Thomas from Centre for Marine Bio-Innovation of the University of New South Wales for the help in the optimization of the analysis pipeline for pyrosequencing datasets and GE Singapore Water Technology Centre for providing the ZeeWeed 500 membrane.

\section{R E F E R E N C E S}

Al Ashhab, A., Herzberg, M., Gillor, O., 2014. Biofouling of reverse-osmosis membranes during tertiary wastewater desalination: microbial community composition. Water Res. 50, 341-349.

Bojsen, R.K., Andersen, K.S., Regenberg, B., 2012. Saccharomyces cerevisiae - a model to uncover molecular mechanisms for yeast biofilm biology. FEMS Immunol. Med. Microbiol. 65 (2), 169-182.

Calderón, K., Rodelas, B., Cabirol, N., González-López, J., Noyola, A., 2011. Analysis of microbial communities developed on the fouling layers of a membrane-coupled anaerobic bioreactor applied to wastewater treatment. Bioresour. Technol. 102 (7), 4618-4627.

Calderón, K., González-Martínez, A., Gómez-Silván, C., Osorio, F., Rodelas, B., González-López, J., 2013. Archaeal diversity in biofilm technologies applied to treat urban and industrial wastewater: recent advances and future prospects. Int. J. Mol. Sci. 14 (9), 18572-18598.

Clarke, K.R., 1993. Non-parametric multivariate analyses of changes in community structure. Aust. J. Ecol. 18 (1), 117-143.

Costello, E.K., Lauber, C.L., Hamady, M., Fierer, N., Gordon, J.I., Knight, R., 2009. Bacterial community variation in human body habitats across space and time. Science 326 (5960), 1694-1697.

Edwards, K.J., Bond, P.L., Gihring, T.M., Banfield, J.F., 2000. An archaeal iron-oxidizing extreme acidophile important in acid mine drainage. Science 287 (5459), 1796-1799.

Fredriksson, N.J., Hermansson, M., Wilén, B.M., 2012. Diversity and dynamics of Archaea in an activated sludge wastewater treatment plant. BMC Microbiol. 12 (1), 140.

Fröls, S., Dyall-Smith, M., Pfeifer, F., 2012. Biofilm formation by haloarchaea. Environ. Microbiol. 14 (12), 3159-3174.

Gomez-Alvarez, V., Revetta, R.P., Domingo, J.W., 2012. Metagenome analyses of corroded concrete wastewater pipe biofilms reveal a complex microbial system. BMC Microbiol. 12 (1), 122.

Gómez-Silván, C., Molina-Muñoz, M., Poyatos, J., Ramos, A., Hontoria, E., Rodelas, B., et al., 2010. Structure of archaeal communities in membrane-bioreactor and submerged-biofilter wastewater treatment plants. Bioresour. Technol. 101 (7), 2096-2105.

Griffiths, R.I., Whiteley, A.S., O’Donnell, A.G., Bailey, M.J., 2000. Rapid method for coextraction of DNA and RNA from natural environments for analysis of ribosomal DNA- and rRNA-based microbial community composition. Appl. Environ. Microbiol. 66 (12), 5488-5491.

Handl, S., Dowd, S.E., Garcia-Mazcorro, J.F., Steiner, J.M., Suchodolski, J.S., 2011. Massive parallel 16S rRNA gene pyrosequencing reveals highly diverse fecal bacterial and fungal communities in healthy dogs and cats. FEMS Microbiol. Ecol. 76 (2), 301-310.

Ivnitsky, H., Katz, I., Minz, D., Volvovic, G., Shimoni, E., Kesselman, E., et al., 2007. Bacterial community composition and structure of biofilms developing on nanofiltration membranes applied to wastewater treatment. Water Res. 41 (17), 3924-3935.

Justice, N.B., Pan, C., Mueller, R., Spaulding, S.E., Shah, V., Sun, C.L., et al., 2012. Heterotrophic archaea contribute to carbon cycling in low-pH, suboxic biofilm communities. Appl. Environ. Microbiol. 78 (23), 8321-8330.

Koerdt, A., Gödeke, J., Berger, J., Thormann, K.M., Albers, S.V., 2010. Crenarchaeal biofilm formation under extreme conditions. PLoS One 5 (11), e14104.

Kolenbrander, P.E., 2000. Oral microbial communities: biofilms, interactions, and genetic systems. Annu. Rev. Microbiol. 54 (1), 413-437.

Kraume, M., Drews, A., 2010. Membrane bioreactors in waste water treatment - status and trends. Chem. Eng. Technol. 33 (8), 1251-1259.

Krysciak, D., Schmeisser, C., Preuß, S., Riethausen, J., Quitschau, M., Grond, S., et al., 2011. Involvement of multiple loci in quorum quenching of autoinducer I molecules in the nitrogen-fixing symbiont Rhizobium (Sinorhizobium) sp. strain NGR234. Appl. Environ. Microbiol. 77 (15), 5089-5099.

Le-Clech, P., Chen, V., Fane, A.G., 2006. Fouling in membrane bioreactors used in wastewater treatment. J. Membr. Sci. 284 (1-2), 17-53.

Li, J., Yang, Y.L., Xue, K., 2013. The applications of MBR in municipal wastewater treatment and reuse. Appl. Mech. Mater. 295-298, 1045-1048.

Lim, S., Kim, S., Yeon, K.M., Sang, B.I., Chun, J., Lee, C.H., 2012. Correlation between microbial community structure and biofouling in a laboratory scale membrane bioreactor with synthetic wastewater. Desalination 287, 209-215.

Macalady, J., Jones, D., Lyon, E., 2007. Extremely acidic, pendulous cave wall biofilms from the Frasassi cave system, Italy. Environ. Microbiol. 9 (6), 1402-1414.

Melin, T., Jefferson, B., Bixio, D., Thoeye, C., De Wilde, W., De Koning, J., van der Graaf, J., Wintgens, T., 2006. Membrane bioreactor technology for wastewater treatment and reuse. Desalination 187 (1-3), 271-282.

Miura, Y., Watanabe, Y., Okabe, S., 2007. Membrane biofouling in pilot-scale membrane bioreactors (MBRs) treating municipal wastewater: impact of biofilm formation. Environ. Sci. Technol. 41 (2), 632-638.

Nguyen, T., Roddick, F.A., Fan, L., 2012. Biofouling of water treatment membranes: a review of the underlying causes, monitoring techniques and control measures. Membranes 2 (4), 804-840.

Orell, A., Fröls, S., Albers, S.V., 2013. Archaeal biofilms: the great unexplored. Annu. Rev. Microbiol. 67 (1), 337-354.

Oren, A., 2001. The bioenergetic basis for the decrease in metabolic diversity at increasing salt concentrations: implications for the functioning of salt lake ecosystems. Hydrobiologia 466 (1-3), 61-72.

Paithankar, K.R., Prasad, K.S.N., 1991. Precipitation of DNA by polyethylene-glycol and ethanol. Nucleic Acids Res. 19 (6), 1346.

Piasecka, A., Souffreau, C., Vandepitte, K., Vanysacker, L., Bilad, R.M., de Bie, T., et al., 2012. Analysis of the microbial community structure in a membrane bioreactor during initial stages of filtration. Biofouling 28 (2), 225-238.

Pruesse, E., Quast, C., Knittel, K., Fuchs, B.M., Ludwig, W., Peplies, J., et al., 2007. SILVA: a comprehensive online resource for quality checked and aligned ribosomal RNA sequence data compatible with ARB. Nucleic Acids Res. 35 (21), 7188-7196.

Ravi, S., Pierce, C., Witt, C., Wormley Jr., F.L., 2009. Biofilm formation by Cryptococcus neoformans under distinct environmental conditions. Mycopathologia 167 (6), 307-314.

Schloss, P.D., Westcott, S.L., Ryabin, T., Hall, J., Hartmann, M., Hollister, E., et al., 2009. Introducing Mothur: open-source, platform-independent, community-supported software for 
describing and comparing microbial communities. Appl. Environ. Microbiol. 75 (23), 7537-7541.

Simões, M., Simões, L.C., Vieira, M.J., 2010. A review of current and emergent biofilm control strategies. LWT — Food Sci. Technol. 43 (4), 573-583.

Song, Z.Q., Wang, F.P., Zhi, X.Y., Chen, J.Q., Zhou, E.M., Liang, F., et al., 2013. Bacterial and archaeal diversities in Yunnan and Tibetan hot springs, China. Environ. Microbiol. 15 (4), $1160-1175$.

Tabatabaei, M., Rahim, R.A., Abdullah, N., Wright, A.D.G., Shirai, Y., Sakai, K., et al., 2010. Importance of the methanogenic archaea populations in anaerobic wastewater treatments. Process Biochem. 45 (8), 1214-1225.

Takai, K., Horikoshi, K., 2000. Rapid detection and quantification of members of the archaeal community by quantitative PCR using fluorogenic probes. Appl. Environ. Microbiol. 66 (11), 5066-5072.
Willett, C.G., Boucher, Y., Di Tomaso, E., Duda, D.G., Munn, L.L., Tong, R.T., et al., 2004. Direct evidence that the VEGF-specific antibody bevacizumab has antivascular effects in human rectal cancer. Nat. Med. 10 (2), 145-147.

Zardus, J.D., Nedved, B.T., Huang, Y., Tran, C., Hadfield, M.G., 2008. Microbial biofilms facilitate adhesion in biofouling invertebrates. Biol. Bull. 214 (1), 91-98.

Zhang, T., Ye, L., Tong, A.H.Y., Shao, M.F., Lok, S., 2011. Ammonia-oxidizing archaea and ammonia-oxidizing bacteria in six full-scale wastewater treatment bioreactors. Appl. Microbiol. Biotechnol. 91 (4), 1215-1225.

Zhang, G.S., Zhang, F., Ding, G., Li, J., Guo, X.P., Zhu, J.X., et al., 2012. Acyl homoserine lactone-based quorum sensing in a methanogenic archaeon. ISME J. 6 (7), 1336-1344. 